$16^{\text {th }}$ International Conference on

AEROSPACE SCIENCES \& AVIATION TECHNOLOGY,

$\boldsymbol{A S A T}$ - 16 - May 26 - 28, 2015, E-Mail: asat@mtc.edu.eg

Military Technical College, Kobry Elkobbah, Cairo, Egypt

Tel : +(202) 24025292 - 24036138, Fax: +(202) 22621908

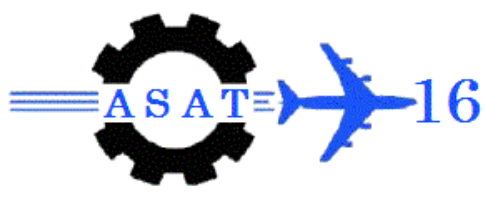

\title{
Development and Analysis of Linear Model Representations of the Quad-Rotor System
}

\begin{abstract}
M. Moness ${ }^{*}$ and M. Bakr ${ }^{\dagger}$
Abstract: A Quad-rotor, which is an airplane with four rotors, is considered a complex and challenging control problem. Analysis and design of its control systems is usually started by careful consideration of its mathematical models description. This paper presents a detailed mathematical model for a Vertical Takeoff and Landing (VTOL) type Unmanned Aerial Vehicle (UAV) known as the quad rotor. The presented paper focus on the nonlinear modeling of an experimental type quad-rotor. The first objective of this paper aims to develop a nonlinear dynamical model based on Newton-Euler approach. Then, a linear model is obtained around an optimized hovering point. The state space, transfer function matrix and matrix fraction description models are derived. Finally, a linear controller is designed to achieve a stable compensated system around a hovering point. The methodology applied in this paper takes the ST-450 experimental quad-rotor as a case study.
\end{abstract}

Keywords: Quad rotor, Modeling, Identification, Linearization, linear control, Stabilization, LQR

\section{Nomenclature}

\begin{tabular}{|c|c|c|c|}
\hline & $\mathrm{I}_{2}$ Quad rotor moment of inertia around $\mathrm{x}$ axis. & $m_{q(\mathrm{~kg})}$ & Mass of quad rotor without battery \\
\hline & Iy Quad rotor moment of inertia around y axis. & $m_{Q(\mathrm{~kg})}$ & Mass of full quad rotor \\
\hline & $\mathrm{I}_{\mathrm{i}}$ Quad rotor moment of inertia around $\mathrm{z}$ axis. & $l_{(m)}$ & Distance to the center of the quad rotor \\
\hline & Jr Single rotor moment of inertia & $g\left(\mathrm{~m} . s^{-2}\right)$ & Gravitational acceleration \\
\hline & $\mathrm{C}$ Thrust factor & & Drag factor \\
\hline UAV & Unmanned aerial vehicles & VTOL & Vertical Takeoff And Landing \\
\hline COG & Center of Gravity & ESC & Electrical Speed Controller \\
\hline 3LDC & Brushless DC Motor & PWM & Pulse Width Modulation \\
\hline DOF & Degree of Freedom & LQR & Linear Quadratic Regulator \\
\hline
\end{tabular}

\footnotetext{
Professor, Computers and Systems Engineering Dept., Faculty of Engineering, Minia University. Email: m.moness@mu.edu.eg.

Teaching assistant, Computers and Systems Engineering Dept., Faculty of Engineering, Minia University. Email: muhammad.bakr@mu.edu.eg.
} 


\section{Introduction.}

UAVs have become progressively popular for commercial and military applications. UAVs and especially quad rotors can be utilized in wide set of real-life applications such as intelligent, surveillance, reconnaissance, wild-fire surveillance, agriculture development, search and rescue, traffic monitoring, pollution air measurements, area exploration and mapping and military applications . Most of the previously declared applications need hovering and VTOL capabilities. In general, fixed-wing aircrafts are incapable to achieve VTOL and be affected from maneuverability limitations. Traditional helicopters are capable of VTOL, but hovering is dynamically and structurally complex, more expensive and hard to control. Quad rotor helicopters are becoming more favorable than helicopters as they are mechanically simpler and easier to control. Although, quad rotor control is a challenging problem, because it is an extremely nonlinear, coupled system, multivariable system, highly unstable nature and underactuated system. Consequently this paper is dedicated to study the problem of modeling the quad copter to get more accurate models, linearization of the derived nonlinear model, and different representation of the linear model and stability analysis.

Modeling of the quad rotor dynamics using a white-box approach. This method assumes full familiarity of both system physical equations and parameters, i.e., it uses a priori information. Amir and Abbas designed and developed mathematical model of a quad rotor dynamics and simplified the quad rotor model using momentum theory. Where the gyroscopic effect and air friction on frame of machine has been neglected [1]. Erginer and Altug studied a white box model for quad rotor, comprised gyroscopic effects for dynamical model and implemented a simulator for the developed model_[2]. Pounds et al constructed and built an X-4 quad rotor then modeling of quad rotor used the basic physics to achieve the mathematical model [3]. Salih et al used Newtonian Methods to describe the dynamic of the quad rotor model [4]. Bouabdallah et al studied the design of quad rotor autonomous robot OS4 and its dynamic nonlinear modelling using Newton- Euler method and vehicle design optimization and control [5]. Bousbaine et al presented a nonlinear dynamic system based on the Euler-Lagrange approach [6].Number of studies has built their linear model based on different linearization techniques. Palm et al designed the open loop linear model of the quad rotor system by linearizing each block in a model individually [7]. Kivrak achieved Linearization of the nonlinear state space equations of the quad rotor system around hovering conditions using Jacobians method [8]. Wilson et al presented a linear state-space method of the quad rotor control system [9]. Ataka et al used the gain scheduling based linearization to achieve linear quad rotor model and simplified the nonlinear model [10].Sørensen used $1^{\text {st }}$ order Taylor approximation to described the appropriate linearization of quad rotor mode [11]. Sonntag presented a linearized model of the quad rotor around hovering condition using identification method [12].

The design of linear controller to achieve a stable compensated system has been studied by many authors. Minh and Cheolkeun designed linear controller for stabilization of the attitude of the quad rotor MAV [13] .Palm et al design control structure that uses feedback to achieve autonomous flight controller [7]. Bouabdallah et al presented the PID and LQ control methods have been reported to successfully stabilize the quad rotor's attitude around hover position in the presence of minor disturbances [14]. Argentim et al presented a comparison between different linear controllers to be used in a dynamic model of a quad copter platform such as PID, LQR and LQR-PID [15]. Lebedev used Pole-Placement state feedback controller design to design a linear controller for stabilization of the quad rotor system [16]. Kivrak used LQR to stabilize of the quad rotor system [8]. Wilson et al presented the linear control of the quad rotor system using pole placement state-feedback controller [9]. Peña et al designed the LQR control with integral effect to track reference paths for the attitude controller [17]. Ataka et al designed linear controller of quad rotor system with LQR state feedback controller [10]. Belkheiri et al presented comparison between different linearization control algorithms such as feedback linearization and LQR [18]. 
Several universities and research group are using quad rotors for scientific research, whether it is a control focused on research or autonomous systems and navigation system. We will show here a brief overview of some of the famous research groups and universities around the world achieved very promising results and used hardware quad rotor. Bouabdallah et al. used a quad rotor model called the OS4 for testing their proposed control algorithms, the OS4 was manufactured and assembled in their laboratory [14]. The X4-Flyer is utilized in the Australian National University [19]. Also, the Mesicopter was developed by Stanford University using off-the-shelf components [19]. Hoffmann et al used the STARMAC II UAV to study the aerodynamic effects on the quad rotor when operating far from the hovering position. Other research groups would rather save the time, cost and effort of building the quad rotor model from scratch and use a commercially available platform. One of the most widely used platform is the one produced by AscTec [20] company. Which is a company that sells quad rotors specially designed to be used in research related tasks. Their prototypes of quad rotor were used by several research groups and universities for example: MIT Robust Robotics Group [21], MIT Aerospace Controls Lab [22] and University of Pennsylvania GRASP Lab [23]. Another widely used platform is the Draganfly from Draganfly Innovations [24] also used by MIT Aerospace Controls Lab [22], [25]. Company is the French company Parrot [26], their model AR.Drone is generally designed for entertainment purposes and it can be remotely controlled by an iPhone mobile. Another company is the Germany Company Microdrones [27] that improves UAVs to be used in various applications for instance aerial surveillance, power lines inspection and bridge inspections among others.

In this paper, we tackle the stabilization and navigation problems of a quad rotor system. A nonlinear model is derived and used for linear controller design. This article is organized as follows: The ST-450 quad rotor kinematics and dynamics models are derived based on the Newtonian mechanics in Section 2. In Section 3, we present the state space model and linear model is obtained around an optimized hovering point. The analysis of our system concerning stability, controllability, observability, minimal realization, System poles and different system representation will be given in section 4. In Section 5, we presented linear state feedback controller to achieve the required performance. The developed simulation framework is introduced in Section 6.Simulation results are presented in Section 7 to show the efficiency of the linear controller. Finally, the paper is concluded in Section 8.

\section{Dynamics modeling of the quad-rotor Model}

Quad rotor helicopter is actuated by four rotors. Four propellers in cross configuration style as shown in Fig.1. Generally, each propeller is directly connected to the Brushless DC Motor (BLDC) motor. Two pairs $(1,3)$ and $(2,4)$, rotating in opposite directions. Input Control forces and moments are generated by changing the speed of the rotors $\left(\omega_{f}, \omega_{l}, \omega_{b}, \omega_{r}\right)$.Variation of rotor speeds to generate Vertical thrust (by concurrent change in rotor speed), pitch moment (by converse change of rotor speeds 1 and 3), Roll moment (by converse change of rotor speeds 4 and 2) and yaw moment (by rotor speed difference between the two pairs of rotors).

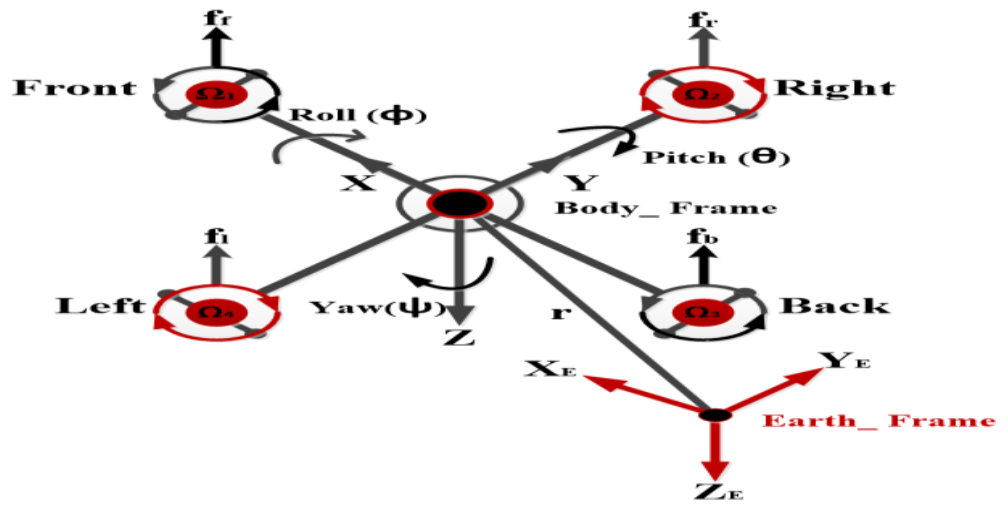

Fig.1 The structure of quad rotor and relative coordinate systems 
In this section, The kinematics and dynamics models of the quad rotor will be derived based on a Newton-Euler formalism with the following assumptions: The quad rotor structure is supposed rigid and symmetrical, the propellers are supposed to be rigid, the cross products of the inertia matrix can be neglected, interaction with ground or other surfaces is neglected and the CoG and the body frame origin are assumed to match. The model of quad rotor consist of three main parts (motor dynamics, rotor velocity to control input and body dynamics). First block, Motor dynamics are include as the first order lag transfer function of 4 BLDC .Secondly، converted actual speed rotor to control input. Third block, body dynamics: as illustrated position $(x, y, \mathrm{z})$ and Euler angles $(\phi, \theta, \psi)$ as illustrated in Fig 2.

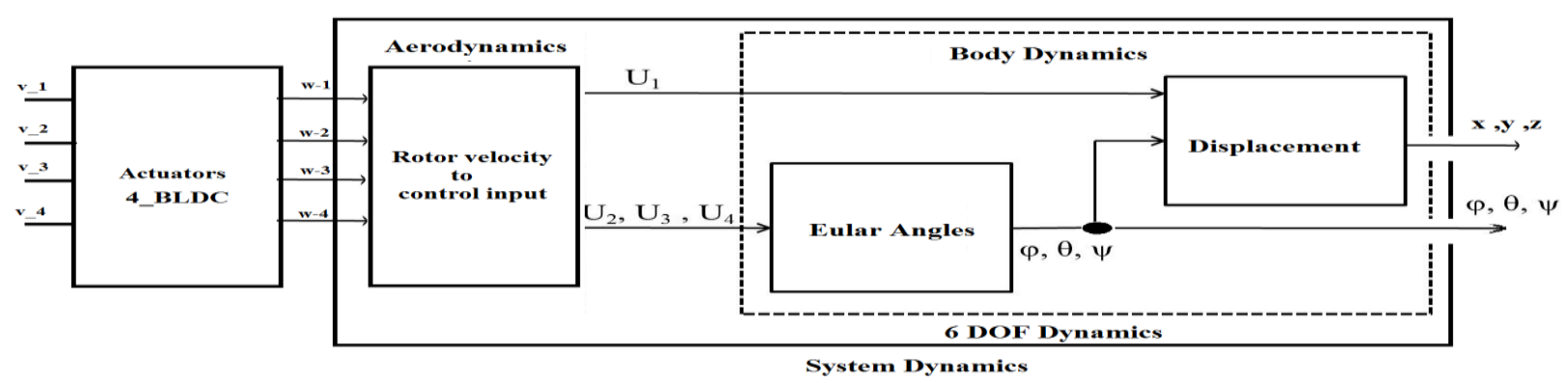

Fig.2 The quad rotor open loop input output block diagram

\subsection{Kinematics Model}

In this part, modelling starts with defining the reference frames .There are two coordinate systems to be considered, the earth frame (E-frame) and the body- frame (B-frame). To transform a vector from the body-frame $(x, y$ and $z)$ to the earth- frame $\left(x_{E}, y_{E}\right.$ and $\left.z_{E}\right)$ shown in fig.1. From the body dynamics, it follows that the two reference frames are connected by the following relations ( [28] , [29]). The following rotational matrix is used [28]

$$
R_{\Theta}=\left[\begin{array}{ccc}
c \psi c \theta & c \psi s \theta s \phi-s \psi c \phi & c \psi s \theta c \phi+s \psi s \phi \\
s \psi c \theta & s \psi s \theta s \phi+c \psi c \phi & s \psi s \theta c \phi-c \psi s \phi \\
-s \theta & c \theta s \phi & c \theta c \phi
\end{array}\right],
$$

$R_{\theta}$ represents the orientation of the aircraft relative to the earth-frame $\left(R_{\theta}=R(\phi, x) R(\theta, y) R(\psi, z)\right.$ is obtained by post-multiplying the three basic rotation matrices. The relation between the dynamics of angular velocity $\left[\begin{array}{lll}p & q & r\end{array}\right]^{\mathrm{T}}$ and the rate of change of the Euler angles $\left(\Phi^{\text {Earth }}=[\dot{\phi} \dot{\theta} \dot{\psi}]^{T}\right)$ can be determined by

As a result, it is likely to describe previous equations in only one equivalence which the relation the derivative of the position in the earth-frame $\left(q^{\text {Earth }}\right)$ to the velocity in the bodyframe ( $\left.q^{\text {body }}\right)$.

\subsection{Dynamics Model}

The Dynamics of the quad rotor system can be divided into two parts, translation subsystem (x, y positions and altitude) and rotational subsystem (roll, pitch and yaw) as shown in fig.2.the translation motion is coupled, while the rotational subsystem is fully decoupled [14] [28]. In the quad rotor relied on the Newton-Euler formulation. 
The Newton-Euler formulation consists of the rotational equations of motion and the translation equations of motion. Firstly, the rotational equations of motion are derived in the body frame using the Newton-Euler method with the following general equation [5]

Where $\tau^{\text {Body }} \in \mathbb{R}^{3 \times 1}$ is the total torque, $I \in \mathbb{R}^{3 \times 3}$ is the diagonal inertia matrix and $\omega^{B o d y}$ angular body rates. Consider the dynamics of the quad rotor, i.e. how the total force and the total torque vectors are composed. Gyroscopic effects are presented if the total sum of the rotational speeds of the rotors $\Omega_{R t o t}=-\Omega_{f}+\Omega_{r}-\Omega_{b}+\Omega_{l}$ is not zero and they influence the total torque adding the following term (see [19], [20]), $\tau_{G r o}^{B o d y}=J_{R o t}[-q p 0]^{T}$. The above obtained relations in one matrix equation the Newton-Euler formulation.

Where $s_{k}(\mathrm{a})$ is the skew-symmetric matrix. While the rotational dynamic model can be written as

$$
[
$$

The aerodynamic moments produced by the $i^{\text {th }}$ rotor are directly proportional to the square of the rotor's speed $M_{i}=C_{i}\left(\omega_{i}^{2}\right)$ where $C_{D}$ drag factor. The aerodynamic moments of the quad rotor in the body-frame are given by

The rotational equations of motion are described by

Neglecting the gyroscopic effects in this case $\Omega_{r}=0$, i.e,

The translation subsystem is based on Newton's second law and they are described in the earth-frame

$n$

Where $F^{\text {Body }} \in \mathbb{R}^{3 \times 1}$ is the total force, $m_{Q}$ is the mass of the quad rotor.

Gravity has effect on the total force $\left(\sum f\right)$ and its direction is along the $\mathrm{z}$ axis of the earthframe. 
The translation dynamic model can be written

\subsection{BLDC Dynamics}

The motors used in quad rotors are BLDC motors that provide little friction and high torque. The dynamics of a BLDC at steady state is the same as a traditional DC motor. In this section, the BLDC model identified experimentally using a system identification tool (e.g. MATLAB's System Identification Toolbox). The system is put on action and the up_down_up is applied to its input as a control command to the BLDC motor. The response of the system to this up_down_up signal is measured. Fig.3. presented the scaled up_down_up step and the scaled response of the system. All inputs and outputs are scaled. The input which represents the control action to the BLDC motor is scaled to the range [25 to 65] that represents [1000 to 7000] RPM. Finally, the first order model was $80.5 \%$ best fit with the reference signal.

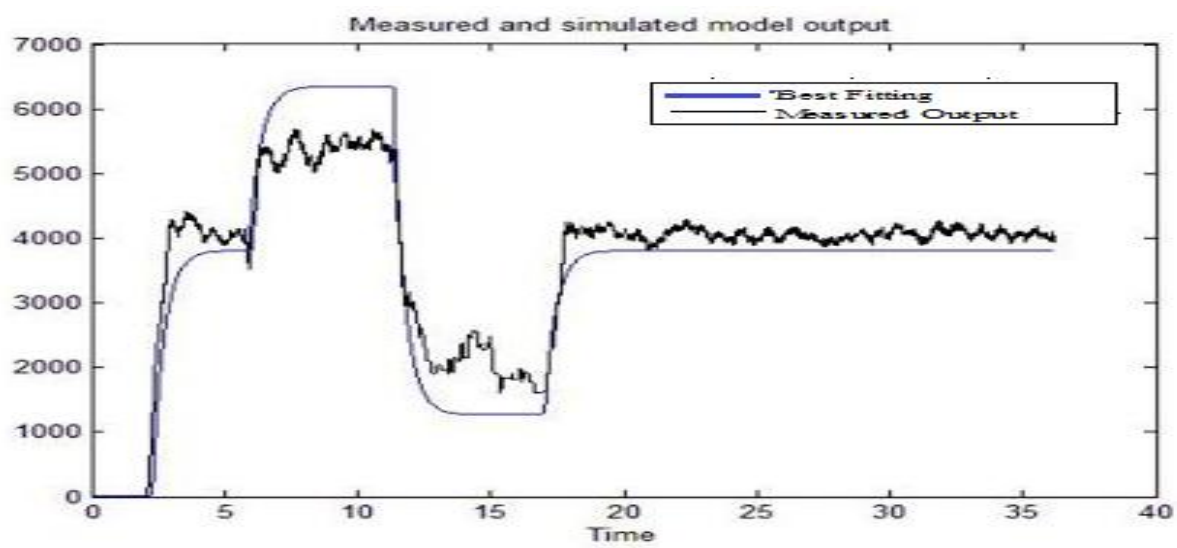

Fig .3. Model Fitting(first order system)

First order lag transfer function was identified to be,

$\mathrm{G}(\mathrm{s})=\frac{\text { Actual Rotor Speed }}{\text { Command Rotor Speed }}=\frac{499.7}{\mathrm{~s}+4.416}$

\subsection{Inertia Calculation}

This part uses the data derived by elementary measurements to identify the body (quad rotor) and rotational moments of inertia. Therefore, the main objective is to derive body moments of inertia matrix I and derive the total rotational moment of inertia around the motor axis

\subsection{Quad Rotor State Space Model}

Defined the state vector of the quad rotor to be

$$
\chi=\left[\begin{array}{llllllllllll}
x_{1} & x_{2} & x_{3} & x_{4} & x_{5} & x_{6} & x_{7} & x_{8} & x_{9} & x_{10} & x_{11} & x_{12}
\end{array}\right]^{T}
$$

Which is mapped to the DOF of the quad rotor as follows.

The state vector defines the quad rotor states. A control input vector, $\mathrm{U}$, consisting of four inputs

$$
U=\left[\begin{array}{llll}
U_{1} & U_{2} & U_{3} & U_{4}
\end{array}\right]^{T} \text {, where }
$$


Using the equations of the rotational angular acceleration and translation Equations 10 and 16 the complete mathematical model of the quad rotor can be written in a nonlinear state space representation as follows

\section{Linearized System}

In this section, the nonlinear quad rotor model described by equation $(10,15)$ can be approximation by the linear model using a first order Taylor's series . The linear approximation of the quad rotor model will be in the form of equation (21).

$$
\left[\Delta \dot{X}_{l}\right]=\left[\frac{\partial f_{j}}{\partial x_{j}}\right]\left[X_{i}\right]+\left[\frac{\partial f_{j}}{\partial u_{k}}\right]\left[\Delta u_{k}\right] \text { For } j, j=1,2,3, \ldots, 12, k=1,2, \ldots, 4
$$

The non-linear model of quad rotor will be linearized at the equilibrium point in order to make the system more amenable. So as to do the linearization, we need to calculate the hovering point of the system. The hovering mode is an operating mode where the quad rotor without motors is stable. The constraints for the hovering operating point are defined in Table 1 .

Table.1. Hovering Point Constraints

\begin{tabular}{|l|c|c|r|r|c|r|r|}
\hline Inputs & Value & Min(RPM) & Max(RPM) & Inputs & Value & Min(RPM) & Max(RPM) \\
\hline & 0 & 1000 & 8000 & & 0 & 1000 & 8000 \\
\hline & 0 & 1000 & 8000 & & 0 & 1000 & 8000 \\
\hline
\end{tabular}

We can get the hovering point by two possible methods, experimentation or optimization by numerical methods of the nonlinear set of differential equations using the previous given constraints Table.1.One problem with the experimentation method that it is quite hard to get the system in hovering by open loop controls, as the system may be marginally stable. Another problem arises with the quantization levels of the inputs that add difficulty to assign precise values that achieve stable hovering. So, it is desirable to calculate the hovering point by numerical optimization method. In this section we will follow optimization method" trustregions" and algorithm "nonlinear least squares trust region reflective". The calculated hovering point data are defined Table 2 .

Table.2. Calculated Hovering Point data

\begin{tabular}{|c|c|c|c|c|c|}
\hline $\begin{array}{c}\text { System } \\
\text { Inputs }\end{array}$ & $\begin{array}{c}\text { Desired Omega } \\
(\text { RPM) }\end{array}$ & $\begin{array}{c}\text { Actual Omega } \\
(\text { RPM) }\end{array}$ & $\begin{array}{c}\text { System } \\
\text { Inputs }\end{array}$ & $\begin{array}{c}\text { Desired Omega } \\
\text { (RPM) }\end{array}$ & $\begin{array}{c}\text { Actual Omega } \\
\text { (RPM) }\end{array}$ \\
\hline & {$[1000,8000]$} & 3134 & & {$[1000,8000]$} & 3134 \\
\hline & {$[1000,8000]$} & 3134 & & {$[1000,8000]$} & 3134 \\
\hline
\end{tabular}

From Equation (22), we can get the linear state space representation as the following equation. 
The state space model of the Quad rotor is approximated using the Simulink Linear Analysis Tool. This tool uses a "Block by block analytic" algorithm, which uses preprogrammed analytic block Jacobians for most blocks and, is more accurate linearization than numerical perturbation of block inputs and states [30].Therefore, the approximated linearized model of quad rotor is described by the following equations.

\section{Characteristics of the ST-450 Quad rotor Linear State Space Model}

In this section, System analysis allows evaluating the characteristics of the future quad rotor in flight or its behavior in various conditions such as stability, construability, Observability and power required.in this part, we will study and analyze the linear quad rotor system.

\subsection{Testing for Controllability and Observability}

Before designing of any controller or any observer, we have to test the controllability and observability of the linear state space system. The controllability matrix for our linear state space system,

The matrix has full rank $\mathrm{n}$, and therefore the system is completely controllable. Similarly the observability matrix.

It has rank $\mathrm{n}$ and therefore it is completely state observable.

\subsection{Minimal Realization}

It is known that realization $\{\mathrm{A}, \mathrm{B}, \mathrm{C}\}$ is minimal if and only if it is controllable and observable. With regard to previous controllability and observability tests, the Quad rotor System has minimal realization.

\subsection{Stability Analysis}

The stability and performance of the proposed linear quad rotor system are analyzed using stability theorem. As the minimal realization of the Quad rotor system linear model has 12 states, then we expect to find 12 poles of the system .To compute the Quad rotor system eigenvalues. The pole characteristic polynomial $\phi(s)$ is defined as: $\phi(s)=\operatorname{det}(s I-A)=$ $\prod_{i=1}^{m}\left(s-p_{i}\right)=0$. All the poles are equal to zero and therefore the system is unstable.

\subsection{Transfer Function Matrix Representations}

Several frequency domain representations of MIMO systems are associated with the same linear time-invariant state space representations. The transfer function matrix $H$ of the state space representation $\{\mathrm{A}, \mathrm{B}, \mathrm{C}, \mathrm{D}\}$ can be defined by $H(s)=C(s I-A)^{-1} B+D$.The $\mathrm{H}(\mathrm{s})$ is returned: 


$$
\left[\begin{array}{cccc}
\frac{-0.2348}{s^{\wedge} 4} & 0 & \frac{0.24}{s^{\wedge}} & 0 \\
0 & \frac{-0.243}{s^{\wedge} 4} & 0 & \frac{0.243}{s^{\wedge} 4} \\
\frac{-0.0016}{s^{\wedge} 2} & \frac{-0.0016}{s^{\wedge} 2} & \frac{-0.0016}{s^{\wedge} 2} & \frac{-0.0016}{s^{\wedge} 2} \\
0 & \frac{-0.0245}{s^{\wedge} 2} & 0 & \frac{0.0245}{s^{\wedge} 2} \\
\frac{0.024}{s^{\wedge} 2} & 0 & \frac{-0.024}{s^{\wedge} 2} & 0 \\
\frac{0.00054}{s^{\wedge} 2} & \frac{-0.00054}{s^{\wedge} 2} & \frac{0.00054}{s^{\wedge} 2} & \frac{-0.00054}{s^{\wedge} 2}
\end{array}\right]=H(s)=\left[\begin{array}{llll}
H_{x 1}(s) & H_{x 2}(s) & H_{x 3}(s) & H_{x 4}(s) \\
H_{y 1}(s) & H_{y 2}(s) & H_{y 3}(s) & H_{y 4}(s) \\
H_{z 1}(s) & H_{z 2}(s) & H_{z 3}(s) & H_{z 4}(s) \\
H_{\phi 1}(s) & H_{\phi 2}(s) & H_{\phi 3}(s) & H_{\phi 4}(s) \\
H_{\theta 1}(s) & H_{\theta 2}(s) & H_{\theta 1}(s) & H_{\theta 4}(s) \\
H_{\psi 1}(s) & H_{\psi 2}(s) & H_{\psi 3}(s) & H_{\psi 4}(s)
\end{array}\right]
$$

\subsection{Rosenbrock System Matrix}

Rosenbrock system matrix is a matrix that groups the four state space matrices $\{A, B, C, D\}$ into a general system matrix. The Rosenbrock System Matrix is defined by Equation (26).

The Rosenbrock system matrix is essentially helpful in extracting system transmission and invariant zeros.

\section{Control system design}

We are now able to design linear controller of the quad rotor system using the matrix $\mathrm{A}, \mathrm{B}$ and $\mathrm{C}$ we have found. The main purpose is to design a full state feedback controller to stabilize the quad rotor system by improving performance of the system using pole placement and Linear Quadratic Regulator methods. Let us verify the controllability of this linearized model of the quad rotor by using state feedback regulator. Constructed equations that will govern the controller dynamics. Since, the state of the system is to be to be feedback as an input, the controller dynamics will be:

$u=r-k x, \dot{x}=A x+B(r-k x), y=C x, \dot{x}=(A-B k) x+B r$

\subsection{Pole placement}

The pole assignment technique is used to place the poles of closed loop system in the desired locations by state feedback. There are two main steps to carry out. First step is the location or assignment of poles and the second step is the calculation of the feedback gain matrix. This approach depends on the performance criteria, such as overshoot $(O S)$, settling time $\left(T_{\text {settling }}\right)$, and rise time used in the design. The design requirements are using time domain specifications to locate dominant poles $s^{2}+2 \zeta \omega_{n} s+w_{n}^{2}=0$. This is done by using the following Equation (29) and finding the dominant poles at $\alpha \pm j \omega_{d}$.

$\zeta=\frac{-\operatorname{In}(O S)}{\sqrt{\pi^{2}+\operatorname{In}^{2}(O S)}}, T_{\text {settling }}=\frac{4}{\zeta \omega_{n}}, \omega_{d}=\omega_{n} \sqrt{1-\zeta^{2}}, \alpha=\zeta \omega_{n}$ and $\omega_{n}=\cos ^{-1} \zeta$

Then placing rest of poles hence they are much faster than the dominant poles.

\subsection{LQR Controller}

Linear quadratic regulator control is a technique to invention optimum solution for a problem of minimization that declares the quad rotor system stability in closed loop. Furthermore, its calculation is very easy the most general problem this approach ca solve is given the dynamic system as Equation (33).the quadratic cost function to minimize:

$\mathbb{J}_{Q R}=\int_{0}^{\infty}\left[x(t)^{T} Q x(t)+u(t)^{T} R u(t)\right]$ $x(t)^{T} Q x(t)$ is the state cost with weight $Q$ $u(t)^{T} R u(t)$ is the control cost with weight $\mathrm{R}$

The matrix $\mathrm{K}$ can be derived from $K=R^{-1} B^{T} P$. While the matrix $\mathrm{P}$ is solution for the algebraic Riccati equation $A^{T} P+P A+Q-P B R^{-1} B^{T} P=0$. The optimal input is defined $u=r-k x$. In order to solve for matrix $\mathrm{K}$, we have to give numerical value so that we get all 
numerical values of matrix $\mathrm{A}$ and $\mathrm{B} . R$ and $Q$ Are weight matrix in order respectively to increase or to decrease the effect of the states. We select small value for weight matrix $\mathrm{R}$ for the reason that we don't need to minimize the energy of input signal. In order to make the quad rotor system able to maintain its altitude, we have to give great amount of energy for input signal. On the other hand, so as to contain the effect of BLDC motor saturation, we contribute maximum and minimum boundary of the motors rotational speed, where $\omega_{\max }=$ $8000 \mathrm{RPM}$ and $\omega_{\min }=1000 \mathrm{RPM}$ according to ST-450 quad rotor model.

\section{Simulation Results}

In this section, we present the simulation result of the controller design and linearization which is presented in previous sections. We have built the ST-450 quad rotor nonlinear model and linear state space model in Matlab/Simulink to test the control algorithms proposed in sections $(5.1,5.2)$ for the quad rotor.as shown in Fig 4. The ST-450 quad rotor parameters used during simulations are shown in Table 3.

Table 3. ST-450 Quad rotor Simulation Parameters

\begin{tabular}{|r|r|r|r|}
\hline$m_{Q}=1.006 \mathrm{~kg}$ & $\mathrm{~g}=9.81 \mathrm{~m} . \mathrm{s}^{2}$ & $\mathrm{C}_{\mathrm{D}=1.9318 e^{-7}}$ & $\mathrm{I}_{\mathrm{yy}}=0.0148$ \\
\hline $\mathrm{l}=0.225 \mathrm{~m}$ & $\mathrm{C}_{\mathrm{T}=2.2901 e^{-5}}$ & $\mathrm{I}_{\mathrm{xx}}=0.0143$ & $\mathrm{I}_{\mathrm{zz}}=0.0246$ \\
\hline
\end{tabular}

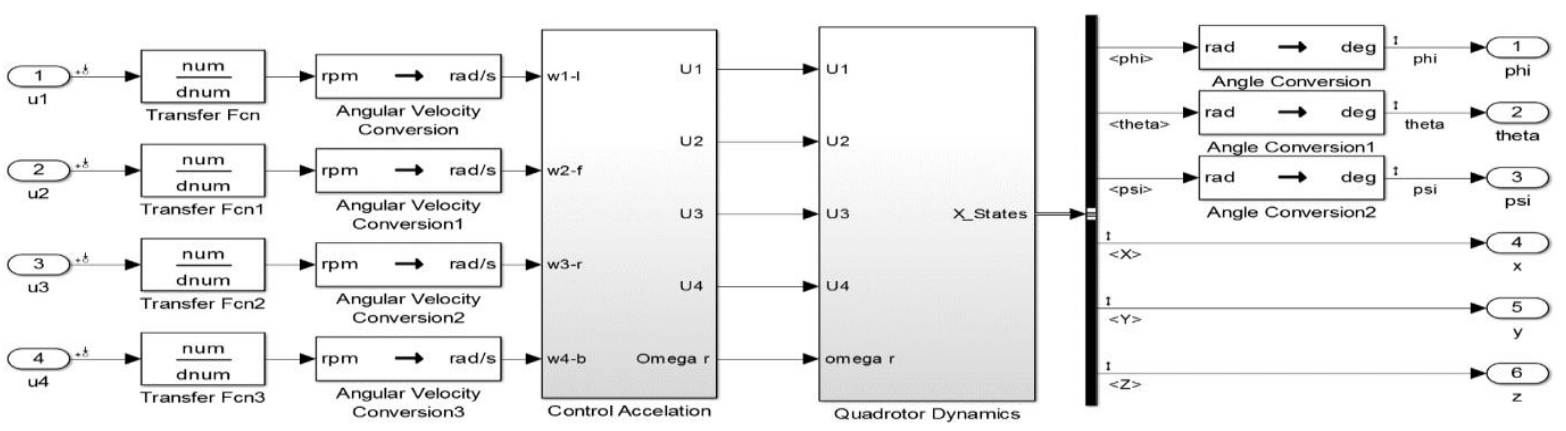

Fig.4.Quad rotor dynamics in MATLAB/SIMULINK

Firstly, the pole placement placing rest of poles hence they are much faster than the dominant poles given $\% O S=0.1$ and $T_{\text {set }}=0.8$, so, the dominant poles are: Dpoles $=5.0000 \pm$ 6.8219i.We can select the desired poles of the system around the dominant poles. Desired poles $=[-5-5.5-5.75-6-6.25-6.5-5.0000 \pm 6.8219 i-6.57-1-2-3]$
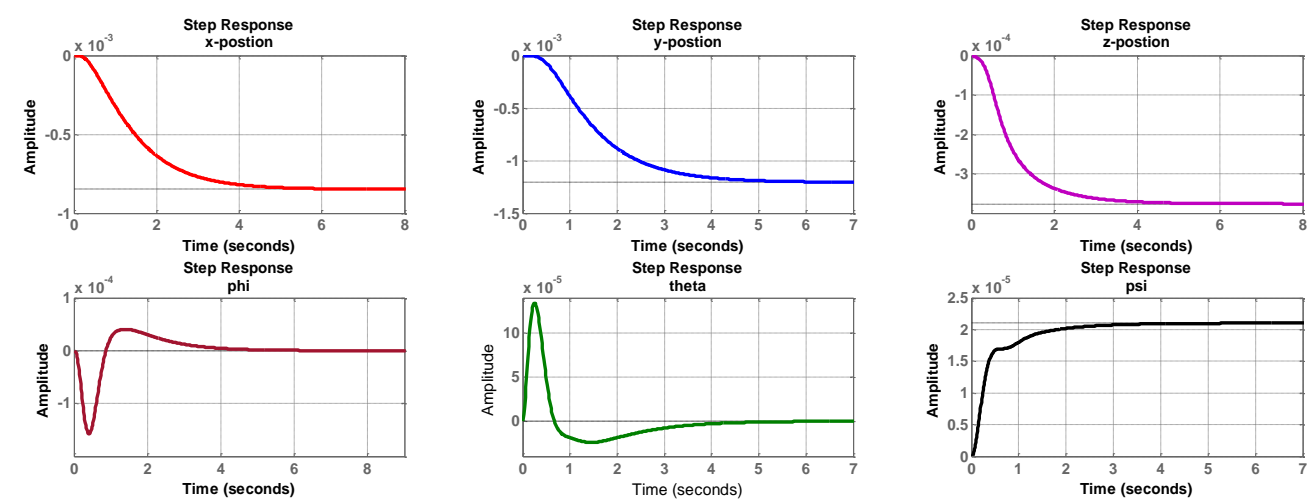

Fig.5. Simulation result of linear state space model in pole placement 
From Fig.5.show the step response of the pole placment controller to stablize the linear quad rotor system .Secondly, LQR then is synthesized using control toolbox to calculate the LQR gains using different weighting $\mathrm{Q}$ and $\mathrm{R}$ matrices., firstly, we can Select design parameter matrices $\mathrm{Q}$ and $\mathrm{R}$ (easily, starting with $Q=C^{\prime} C, R=1$.Secondly, Selecting design parameter matrices $\mathrm{Q}$ and $\mathrm{R}\left(Q=q I_{12} \times 12, R=r I_{4 \times 4}\right.$. where $\mathrm{q}=10$ and $\mathrm{r}=0.1$. This is done supposing the model is described in the following equations $(35,36)$ and is presented in Fig 6. Thirdly, Selecting design parameter matrices $\mathrm{Q}$ and $\mathrm{R}\left(Q=q I_{12 \times 12}, R=\right.$ $r I_{4 \times 4}$.where $\mathrm{q}=100$ and $\mathrm{r}=0.01$. This is done supposing the model is described in the following equations $(37,38)$ and is presented in Fig 7.

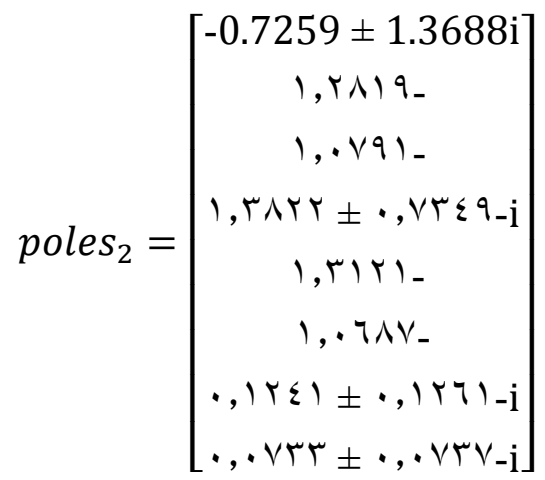

$K_{L Q R 3}=$
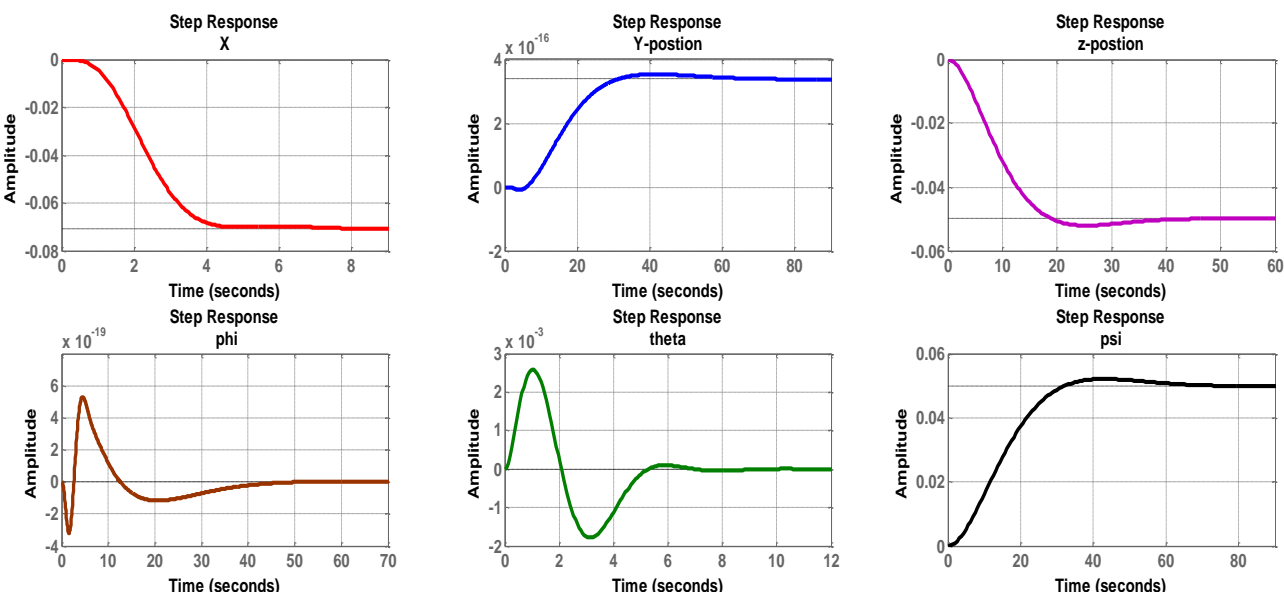

Fig.7. Simulation result of linear state space model in $L Q R_{2}$ 

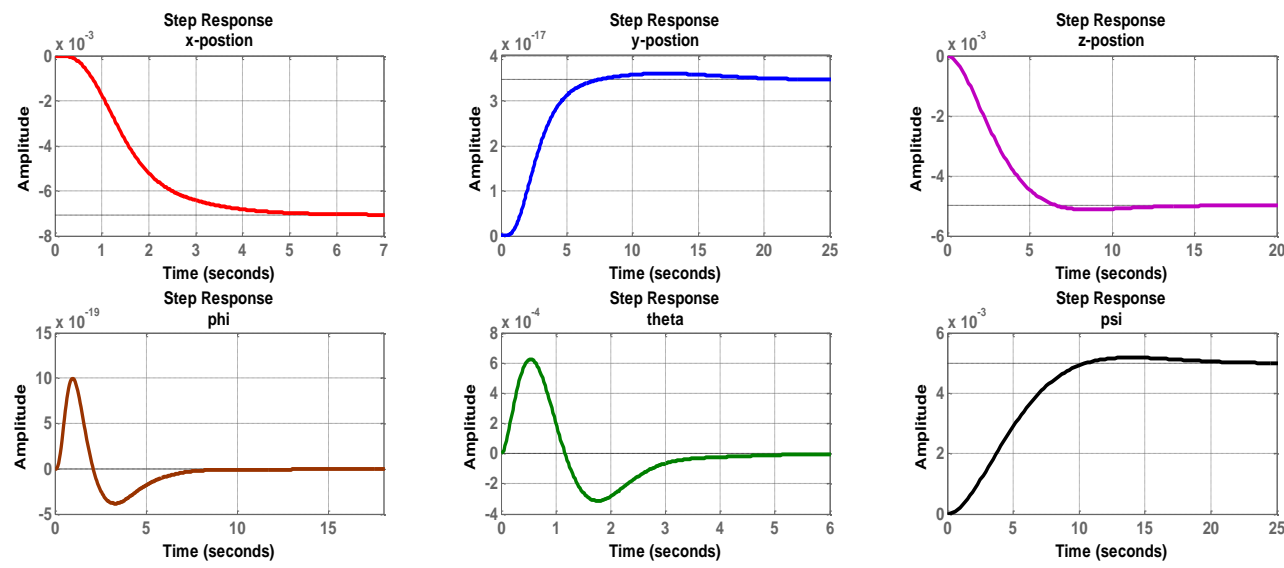

Fig.8. Simulation result of linear state space model in $L Q R_{3}$

According to system step response results, $L Q R_{3}$ has an acceptable command following capability with reasonable settling time, rise time and overshoot along with $L Q R_{2}$, but the pole placement method has faster settling time than $L Q R_{3}$ and $L Q R_{2}$.

\section{Conclusion}

In this article, we get the linearized model of quad rotor simplified model and show that the system is controllable and observable regardless of the value of reference position. The model of quad rotor system based on Newtonian mechanics was developed. The BLDC motor of the model were identified using Identification toolbox. Parameters of the model were calculated using a test based on an approach of successive measurements. The nonlinear model is first achieved and then represented by a state space model an approximated linear model of the Quad-rotor system was extracted using a first-order Taylor's series. We use the simplified model of quad rotor based on ST-450 model then linearize it to get the linearized state equation at some hovering point. Then, we investigation the controllability, observability, mineralization and stability of the linear model of the quad rotor system and prove that the system is controllable and observable regardless of the value of the operating point we choose. Then, we check the linear quad rotor system behavior by using the state feedback controller and designed a state feedback controller using pole placement and LQR. Simulation results are carried out to show that the designed linear controllers make sure the convergence of the error dynamics in closed-loop system. Some simulations in the presence of model parametric insecurity and some external disturbance forces will be included to complete this research. We get from this article show that the simplified model of ST-450 quad rotor can be controlled by more advanced linear controller method such as LQR and pole placement and we used this controllers to stabilize the linear quad rotor system. All controllers have acceptable suppressing capabilities for the linear model. Examinig results, it is clear that pole placement has the least overshoot and acceptable settling time, therefore it is considered the best controller compared with other controllers. Finally, we need to increase the efficiency of each controller. We will redesign these controllers using heuristic tuning algorithm of the state feedback controller or we will design another controller such as PID controller and sliding mode controller.

\section{Reference}

[1] M. Amir and V. Abbass, "Modeling of Quadrotor Helicopter Dynamics," in Smart Manufacturing Application, 2008. ICSMA 2008. International Conference on, Gyeonggi-do, 9-11 April 2008.

[2] B. Erginer and E. Altuð, "Modeling and PD Control of a Quadrotor VTOL Vehicle," in IEEE Intelligent Vehicles Symposium, Istanbul, Turkey, June 13-15, 2007.

[3] P. Pounds, R. Mahony and P. Corke, "Modelling and Control of a Quad-Rotor Robot," Control Engineering Practice, vol. 18, no. 7, pp. 691-699, 2010.

[4] A. L. Salih, M. Moghavvemi, H. A. F. Mohamed and K. S. Gaeid, "Modelling and PID controller 
design for a quadrotor unmanned air vehicle," in Automation Quality and Testing Robotics (AQTR), 2010 IEEE International Conference on (Volume:1), Cluj-Napoca, 28-30 May 2010.

[5] S. Bouabdallah, P. Murrieri and R. Siegwart, "Design and control of an indoor micro quadrotor," in Robotics and Automation, 2004. Proceedings. ICRA '04. 2004 IEEE International Conference on, Switzerland, 26 April-1 May 2004.

[6] A. Bousbaine, M. H. Wu and G. T. Poyi, "Modelling and simulation of a quad-rotor helicopter," in Power Electronics, Machines and Drives (PEMD 2012), 6th IET International Conference, Bristol, 27-29 March 2012.

[7] J. Palm, A. Nelson and A. Bradford, "Quad-Rotor UAV project „Final Report," DSSL Lab, Virginia Tech, June 11, 2010.

[8] A. Ö. KIVRAK, "A MASTER'S THESIS,DESIGN OF CONTROL SYSTEMS FOR A QUADROTOR FLIGHT VEHICLE EQUIPPED WITH INERTIAL SENSORS," Mechatronics Engineering ATILIM UNIVERSITY, DECEMBER 2006.

[9] G. N. Wilson, A. Ramirez-Serrano and a. Q. Sun, "Vehicle Parameter Independent Gain Matrix Selection for a Quadrotor using State-Space Controller Design Methods," in 11th International Conference on Informatics in Control, Automation and Robotics, Vienna, Austria, September 1-3, 2014.

[10] A. T. H. Ataka, R. Inovan, M. Abdurrohman, H. Preastianto, A. Cahyadi and Y. Yamamoto, "Controllability and Observability Analysis of theGain Scheduling Based Linearization for UAV Quadrotor," in 2013 International Conference on Robotics, Biomimetics, Intelligent Computational Systems (ROBIONETICS), Yogyakarta, Indonesia, November 25-27,2013.

[11] A. F. Sørensen, "Autonomous Control of a Miniature Quadrotor Following Fast Trajectories,Master's Thesis," June 3rd, 2010.

[12] D. Sonntag, "A Study of Quadrotor Modelling,Final Thesis," IDA, Linköpings University, Linköping, Sweden, 2011-03-18.

[13] L. D. Minh and C. Ha, "Modeling and Control of Quadrotor MAV Using Vision-based Measurement," in Strategic Technology (IFOST), 2010 International Forum on, Ulsan, 13-15 Oct. 2010.

[14] S. Bouabdallah, A. Noth and R. Siegwan, "PID vs LQ Control Techniques Applied to an Indoor Micro Quadrotor," in Proceedings of 2004 IEEEIRS.J Internationel Conference On Intelligent Robots and Systems, Sendal, Japan, September 28. October 2,2004.

[15] L. M. Argentim, W. C. Rezende, P. E. Santos and R. A. Aguiar, "PID, LQR and LQR-PID on a Quadcopter Platform," in Informatics, Electronics \& Vision (ICIEV), 2013 International Conference on, Dhaka, 17-18 May 2013.

[16] A. Lebedev, "Design and Implementation of a 6DOF Control System for an Autonomous Quadrocopter,Master Thesis," Aerospace Information Technology' Department, University of Würzburg, 07- 09- 2013.

[17] M. V. P. G., E. C. V. G. and C. I. R. F., "SIMULATION OF THE QUADROTOR CONTROLLED WITH LQR WITH INTEGRAL EFFECT," in ABCM Symposium Series in Mechatronics - Vol. 5, 2012.

[18] M. BELKHEIRI, A. RABHI, A. E. HAJJAJI and C. P'EGARD, "Different linearization control techniques for a quadrotor system," in Communications, Computing and Control Applications (CCCA), 2012 2nd International Conference on, Marseilles, 6-8 Dec. 2012.

[19] J. Jun, Q. Juntong, S. Dalei and H. Jianda, "Control platform design and experiment of a quadrotor," in Control Conference (CCC), 2013 32nd Chinese, Xi'an, 26-28 July 2013.

[20] Ascending Technologies, [Online]. Available: http://www.asctec.de/uavapplications/research/products/. [Accessed 16 April 2015].

[21] MIT Robust Robotics Group, [Online]. Available: http://groups.csail.mit.edu/rrg/.. [Accessed 16 April 2015].

[22] MIT Aerospace Controls Lab., [Online]. Available: http://acl.mit.edu/.. [Accessed 16 April 2015].

[23] University of Pennsylvania GRASP Lab., [Online]. Available: https://www.grasp.upenn.edu/. [Accessed 16 April 2015]. 
[24] Dragan-Fly, [Online]. Available: http://www.draganfly.com/. [Accessed 16 April 2015].

[25] F. Kendoul, "Survey of advances in guidance, navigation, and control of unmanned rotorcraft systems," Journal of Field Robotics, vol. 29, no. 2, pp. 315-378, 2012.

[26] Parrot AR Drone, [Online]. Available: http://ardrone2.parrot.com/. [Accessed 15 April 2015].

[27] Microdrones Germany Company , [Online]. Available: http://www.microdrones.com/en/home/. [Accessed 16 April 2015].

[28] T. Bresciani, "Modelling, Identification and Control of a Quad rotor Helicopter,MASTER THESIS," Lund University, Department of Automatic Control, Lund Sweden, October 2008.

[29] P. E. I. Pounds, "Design, Construction and Control of a Large Quadrotor Micro Air Vehicle,Doctor of Philosophy," Australian National University, Austra, September 2007.

[30] T. B. CO, in Method of applied Mathematics for engineers and scientists, pp. Ch. 8: P.311-340. 